\section{FRI0520 THE HUMAN ENTHESIS CONTAINS POPULATIONS OF MESENCHYMAL STEM CELLS WITH DISTINCT FUNCTIONAL CHARACTERISTICS}

Tobias Russell $^{1}$, Abdulla Watad ${ }^{2}$, Charlie Bridgewood ${ }^{1}$, Almas Khan ${ }^{3}$, Peter Millner ${ }^{3}$, Peter Loughenbury ${ }^{3}$, Abhay S. Rao ${ }^{3}$, Robert Dunsmuir ${ }^{3}$, Thomas Baboolal' ${ }^{1}$, Elena Jones ${ }^{1}$, Dennis Mcgonagle ${ }^{1}$, Richard Cuthbert ${ }^{1}$. ${ }^{1}$ University of Leeds, Leeds Institutute of Rheumatic and Musculoskeletal Medicine, Leeds, United Kingdom; ${ }^{2}$ Sheba Medical Center, Internal Medicine B, Ramat Gan, Israel; ${ }^{3}$ Leeds Teaching Hospitals NHS Trust, Leeds, United Kingdom

Background: Ankylosing spondylitis (AS) is associated with entheseal inflammation and new bone formation. Resident populations of lymphocytes have been identified at the enthesis that on stimulation with IL-23 produce pro-inflammatory cytokines including IL-17A and IL-22 (1) which drive inflammation and may also influence osteogenesis. Surprisingly, enthesis resident mesenchymal stem cells (MSCs) have not been phenotypically or functionally characterised.

Objectives: To determine if human enthesis including entheseal soft tissue and peri-entheseal bone harbours a population of MSCs. Furthermore, to investigate the effect of spondyloarthritis associated proinflammatory cytokines on MSC osteogenesis.

Methods: Samples from healthy the spinous process and interspinous ligament $($ male $=5$, female $=10$, median age $=19)$ were divided into entheseal soft tissue (EST) and peri-entheseal bone (PEB) and enzymatically digested (1). MSCs content was assessed using a CFU-F assay. Flow cytometry was used to examine expression of MSCs specific markers in plastic adherent cultures. Following osteogenic, chondrogenic and adipogenic inductions, osteogenesis was qualitatively by alkaline phosphatase and alizarin red staining and quantitatively by measurement of calcium accumulation. Chondrogenesis and adipogenesis were assessed using glycosaminoglycan assay and oil red o staining respectively. Osteogenic cultures were also supplemented with IL-17A $(50 \mathrm{ng} / \mathrm{ml})$, IL-22 (10ng/ml) or TNF- $\alpha(1 \mathrm{ng} / \mathrm{ml})$.

Results: As a proportion of total cellularity EST developed approximately 5 fold more colonies than matched PEB $(p<0.0001)$. Cultured cells were overwhelmingly positive for expression of MSC markers CD73, CD90, CD105 (PEB median $98.66 \%$ range: $95.17-98.96 \%$, EST median $98.42 \%$ range: $87.69-98.7 \%$ ) and negative for CD14, CD19, CD45 and HLA-DR (PEB median $0.34 \%$ range: $0-2.91 \%$, EST median $1.1 \%$ range: $0-2.53 \%$ ) however some CD34 expression was noted particularly in EST cultures (median $3.74 \%$ range: $0-29 \%$ ). Both populations were capable of tri-lineage differentiation, although PEB MSCs had greater osteogenic $(p<0.025)$ and lower adipogenic $(p<0.05)$ potential than matched EST MSCs. Addition of IL-22 $(p<0.01)$ or IL-17A $(p<0.005)$ caused a significant decrease in the calcium deposited in PEB MSCs but not in EST MSCs.

Conclusion: Both the EST and PEB contain cells that meet the ISCT criteria defining MSCs. However, MSCs from these sources are functionally distinct in terms of their differentiation potential and response to inflammatory cytokines. The cytokines tested had a negative influence on osteogenesis in the conditions tested.

\section{REFERENCES:}

[1] CUTHBERT, R.J., E.M. FRAGKAKIS, R. DUNSMUIR, Z. LI, M. COLES, H. MARZO-ORTEGA, P.V. GIANNOUDIS, E. JONES, Y.M. EL-SHERBINY and D. MCGONAGLE. Brief Report: Group 3 Innate Lymphoid Cells in Human Enthesis. Arthritis Rheumatol, 2017, 69(9), pp.1816-1822.

Disclosure of Interests: Tobias Russell Grant/research support from: PhD Project is funded by Novartis., Abdulla Watad: None declared, Charlie Bridgewood: None declared, Almas Khan: None declared, Peter Millner: None declared, Peter Loughenbury: None declared, Abhay S Rao: None declared, Robert Dunsmuir: None declared, Thomas Baboolal: None declared, Elena Jones: None declared, Dennis McGonagle Consultant for: Lilly, Novartis UCB, Speakers bureau: Lilly, Novartis UCB, Richard Cuthbert: None declared

DOI: 10.1136/annrheumdis-2019-eular.2638

\section{FRI0521 MIRNA-150 AS A NOVEL REGULATOR OF POST- TRAUMATIC OSTEOARTHRITIS}

Asaad Al Adlaan ${ }^{1}$, Bryson Cook ${ }^{1}$, Fatima Jaber ${ }^{1}$, Fayez Safadi ${ }^{1,2} .{ }^{1}$ Rootstown, Anatomy and Neurobiology, Musculoskeletal Research Group, Rootstown, United States of America; ${ }^{2}$ Akron, Rebecca D Considine Research Institute, Akron Children's Hospital, Akron, United States of America

Background: Osteoarthritis $(\mathrm{OA})$ is a chronic joint disease causes irreversible damage to the articular cartilage resulting in loss of joint function and subchondral bone remodeling. There is no cure for OA and currently the available treatment like pain management, and joint replacement surgery is used to treat this disease. MiRNAs are small non-coding RNAs consist of 21-29 nucleotides which regulate gene expression by targeting the 3' untranslated region (UTR) leading to translation inhibition. In recent years, many studies showed that microRNAs could be a target for many diseases including OA. Our preliminary data on osteoactivin showed that the GPNMB acts as a protective protein in osteoarthritis by reducing catabolic gene expression. Our lab group results have been shown that miRNA-150 targets the 3'UTR of osteoactivin in osteoblast cells.

Objectives: There is no study regarding the role of miRNA 150 on cartilage homeostasis and because the miRNA 150 targets osteoactivin in lung and bone cells, we hypothesized that miRNA-150 targets osteoactivin in chondrocytes and protect cartilage ECM degradation.

Methods: RNAs were isolated from primary chondrocytes of miRNA- 150 knockout pups, then was used in $\mathrm{qPCR}$ to measure gene expression. Western blot used to detect MMP-3, MMP-9, MMP-13, IL-6, collagen type-II and aggrecan. In addition, histological analysis was used to test the role of miRNA-150 vivo through evaluating proteoglycan degradation by toluidine blue staining and immunohistochemistry after 12 weeks from DMM surgery. PCR transcription factors and signaling pathway finder array were used to elucidate the altered genes and transcriptions factors compared to the wild type in primary chondrocytes culture.

Results: Primary chondrocytes from $150 \mathrm{KO}$ mice showed high mRNA expression levels of GPNMB compared to the wild type mice. Medial tibia plateau (MTP) and medial femur condyle (MFC) have been graded by OARSI scoring system after 12 weeks of DMM surgery. Histological results showed that the $150 \mathrm{KO}$ mice have less cartilage damaged compared to the wild type. To determine whether epigenetic modifiers (miRNAs) regulating GPNMB/Osteoactivin expression can modulate the cartilage protective effects of GPNMB/Osteoactivin, we found that miRNA 150 targets GPNMB/Osteoactivin in chondrocytes. Next, we examined the effects of miRNA 150 deficiency on the OA progression in vivo and found that miRNA $150 \mathrm{KO}$ are more protected against post-traumatic OA compared to WT mice. Furthermore, we assessed the expression of GPNMB/Osteoactivin in miRNA $150 \mathrm{KO}$ chondrocytes and showed a significant increase compared to WT cells.

Conclusion: These results showed that miRNA is negative regulator of cartilage homeostasis and blocking miRNA might have therapeutic potential fort he treatment of age-related osteoarthritis and post-traumatic induced osteoarthritis.

\section{REFERENCES:}

The surgical destabilization of the medial meniscus (DMM) model of osteoarthritis in the 129/SvEv mouse. Methods in Molecular Biology: Osteoporosis and Osteoarthritis. Loeser RF, Goldring SR, Scanzello CR, Goldring MB. Osteoarthritis: A disease of the joint as an organ. Arthritis and Rheumatism. 2012. pp. 1697-1707.

[1] Zhou, L., Zhuo, H., Ouyang, H., Liu, Y., Yuan, F., Sun, L.,. .. \& Liu, H. (2017). Glycoprotein non-metastatic melanoma protein b (Gpnmb) is highly expressed in macrophages of acute injured kidney and promotes M2 macrophages polarization. Cellular immunology, 316, 53-60.

[2] Ripoll, V.M., et al., Gpnmb is induced in macrophages by IFN-gamma and lipopolysaccharide and acts as a feedback regulator of proinflammatory responses. J Immunol, 2007. 178(10): p. 655766.

Acknowledgement: This research was funded by the Cook Research Fund and Ohio Department of Education.

Disclosure of Interests: None declared

DOI: 10.1136/annrheumdis-2019-eular.7178 